\title{
Intelligent road traffic management based on the system of fuzzy situational management and virtual cyberspace
}

\author{
Sergey Korjagin ${ }^{1}$, Ksenia Polupan $^{1}$, Pavel Klachek $^{1 *}$, Alexey Pyatikop ${ }^{1}$, and Evgeniy \\ Koryagin $^{1}$ \\ ${ }^{1}$ Immanuel Kant Baltic Federal University, 236016 Kaliningrad, Russia
}

\begin{abstract}
Theoretical and applied ideas and tools of traffic intelligent management on the basis of fuzzy situational management and virtual cyberspace are developed through the system approach, artificial intelligence methods, modern achievements in the field of the creation of intelligent transport systems (ITS). The proposed scientifically-methodical foundations and software and hardware tools allow the creation of intelligent transport systems at a new level, synchronizing the development of road traffic infrastructure and virtual cyberspace, allowing to solve effectively a rather large range of theoretical and applied problems in the field of traffic management, transport modelling and planning.
\end{abstract}

\section{Introduction}

Development of science-based methodologies and tools $[2,9-12,14,15]$ aimed at conducting in-depth surveys in the field of transport infrastructures, followed by the implementation of management using intelligent transport systems (ITS) is one of the promising areas in the development of efficient urban transport systems. On the other hand, it is necessary to develop approaches and tools $[3,12,15]$ allowing to combine at a new level technologies of mathematical modeling of transport processes, real-time management, decision support systems, communication technologies, etc $[18,19]$.

The work [2, 3] presents the author's approach in the field of creation of adaptive intelligent transport systems of new generation based on application of fuzzy situational management and virtual cyberspace. Applied intelligent systems of transport processes management of various types and purposes, created on the basis of the proposed approach, will allow not only solving the classical tasks specific to ITS (monitoring of traffic characteristics, traffic flow (TF) adaptive management, etc.) but also providing the scientific basis and applied tools for perspective directions in the field of creating intelligent transport infrastructures, based on the solution of the following tasks:

- Intellectualization of the vehicle itself, its transformation into the most automated and adaptive autonomous system $[2,3,16]$;

* Corresponding author: pklachek@mail.ru 
- Creation of mathematical and applied tools for information interaction between individual vehicles with the purpose of coordination and optimization of their group behavior as separate interconnected subsystems of a complex system, which gives the transport system the properties of a single information environment [1,2].

- Creation of mathematical and applied tools that can provide a synergetic effect of selforganization and development of the transport system, resulting from the interaction of individual cognitive (accumulating knowledge) subsystems into a single system, subordinated to a single strategic goal and interacting according to the laws of coalition behavior [1].

Thus, the problem of developing theoretical foundations of intelligent traffic management based on fuzzy situation management and virtual cyberspace, allowing to ensure at a new level the creation of highly adaptive intelligent transport systems [17], synchronizing the development of road transport infrastructures and virtual cyberspace, allowing to solve effectively a fairly large range of theoretical and applied problems in the field of traffic management, transport modeling and planning on the basis of use of diverse knowledge, is relevant and timely.

\section{Material and methods}

In [2-4] a hybrid model of TF management is presented in detail. The central element of the model is a fuzzy system of the form:

$$
s=\left\{\left\langle\mu_{s}\left(y_{i}\right) / y_{i}\right\rangle\right\}, y_{i} \in Y,
$$

where, $\mu_{s}\left(y_{i}\right)$ - is the function of belonging to a linguistic variable (the diflex factor of TF [25]) $y_{i}$, where $i-$ is the number of TF variable. Each TF is represented by a set of variables [2-4]: intensity, speed, type, flow interval, etc., for each of which a two-parameter hyperbolic approximation form is calculated [3-5], (fig.1).

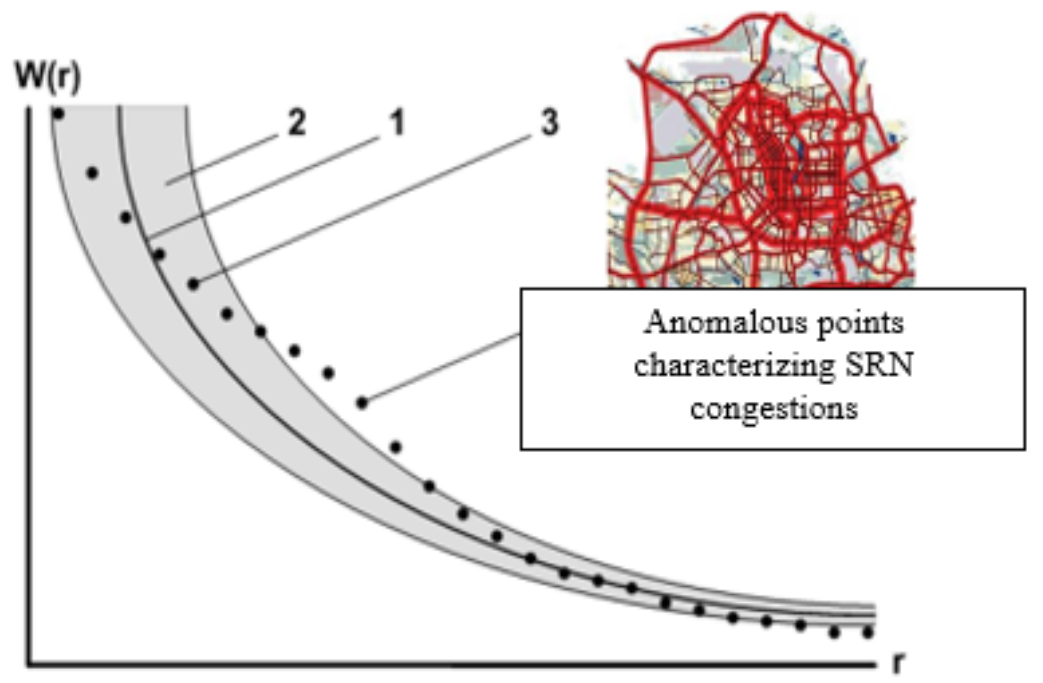

Fig. 1. Calculation of anomalous points and the diflex parameter of the street-road network objects: 1 - approximating curve; 2 - variable confidence interval; 3 - empirical points of rank distribution.

where, $W(r)$ - is the value of the investigated parameter (for example, traffic flow intensity) of the street-road network (SRN) object with r-rank; $W_{1}-$ is the value of the investigated object parameter of rank one (the object with the maximum value of the investigated 
parameter); $\beta$ - is the rank coefficient characterizing the shape of the $H$-distribution curve [4, 5].

Further, in accordance with the GZ-analysis, which is described in detail in [3, 4], anomalous points are determined and the diflex parameter of the road network objects is calculated (Fig. 1,2):

$$
\Delta W_{k}^{i}=\frac{W_{k}-W_{\left(r_{k}\right)}^{B}}{W_{k}} .
$$

Where: is the empiric value of the k-object parameter of the SRN, $W^{B}{ }_{(r k)}-$ is the value of the parameter corresponding to the k-rank at the upper boundary of the variable confidence interval of the rank distribution. $i-$ is the number of the TF variable.

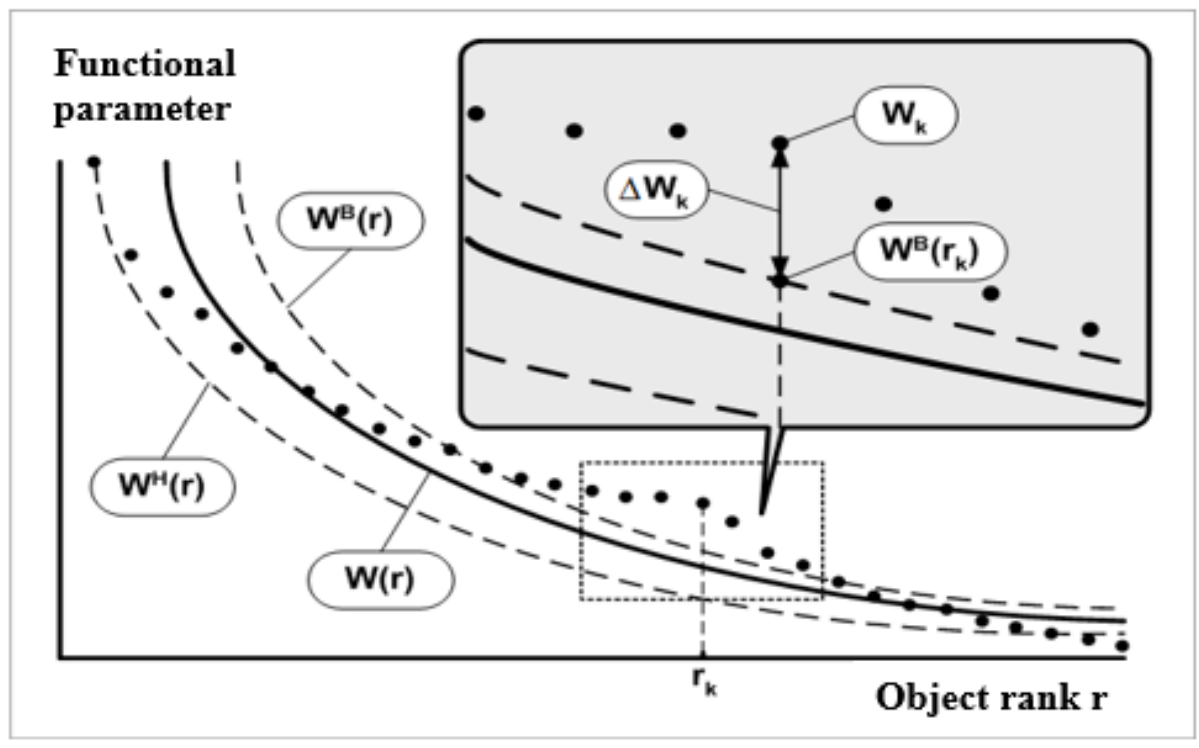

Fig. 2. Determination of the diflex parameter of the street-road network object.

Each diflex-parameter is assigned with the membership function of the linguistic variable $y_{i}$ and the corresponding set of term sets [4].

Transport process management is carried out on the basis of a fuzzy intelligent semantic network (see Fig.3) presented in detail in [6]. It constitutes a fuzzy semantic graph, vertices $s_{i}$ of which correspond to fuzzy transport situations, and semantic $\operatorname{arcs} R_{j}$ - to managing solutions.

Thus, the development of the solution is divided into the goal setting (goal situation) and the construction of a management strategy corresponding to the optimal transfer of the object to the target state.

Studies [2-4] show that the proposed approach allows smart and elegant (including in terms of computing time consumption) implementation and analysis of the relationship between the main TF parameters (speed, intensity, density) on many levels, investigation of $\mathrm{TF}$ behavior at critical points - saturation and congestion, and also effective solution to a rather large range of theoretical and applied problems in the field of traffic management, transport modeling and planning. 


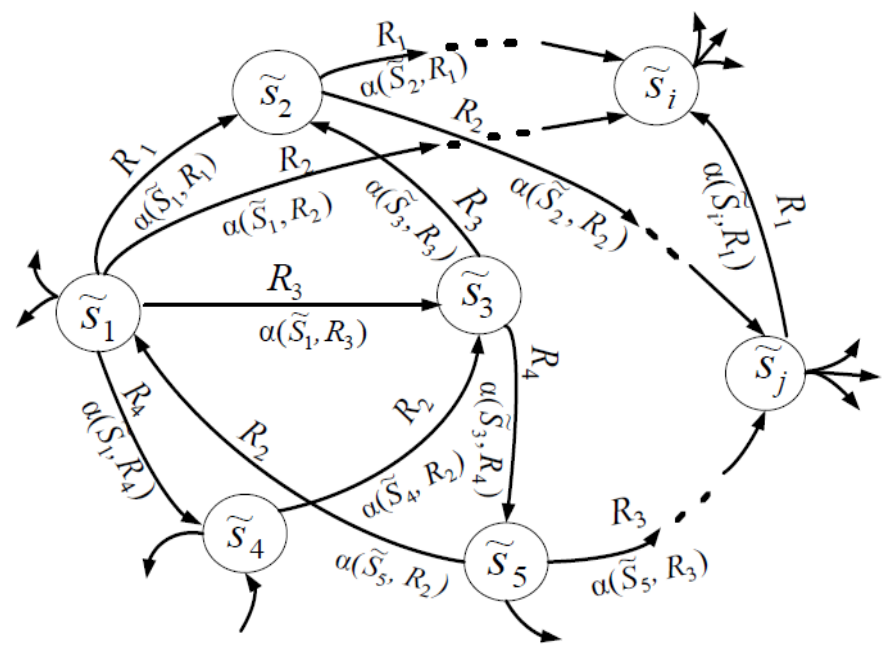

Fig. 3. Fuzzy intelligent semantic network.

\section{Theory}

The above approach of fuzzy situation management in solving traffic management problems makes special demands on infrastructure monitoring and intelligent traffic management. In [6] a basis was proposed, and in [2-4, 7, 8] the ITS perceptual infrastructure platform was developed (Fig. 4), in which the ITS adaptive control center [Klachek et al. (2015)] is integrated into "cloud", and operates on the basis of frame-services of virtual cyberspace [3, $4,6]$, accessible through a wireless global communication network having a distributed structure and accessible all over the world.

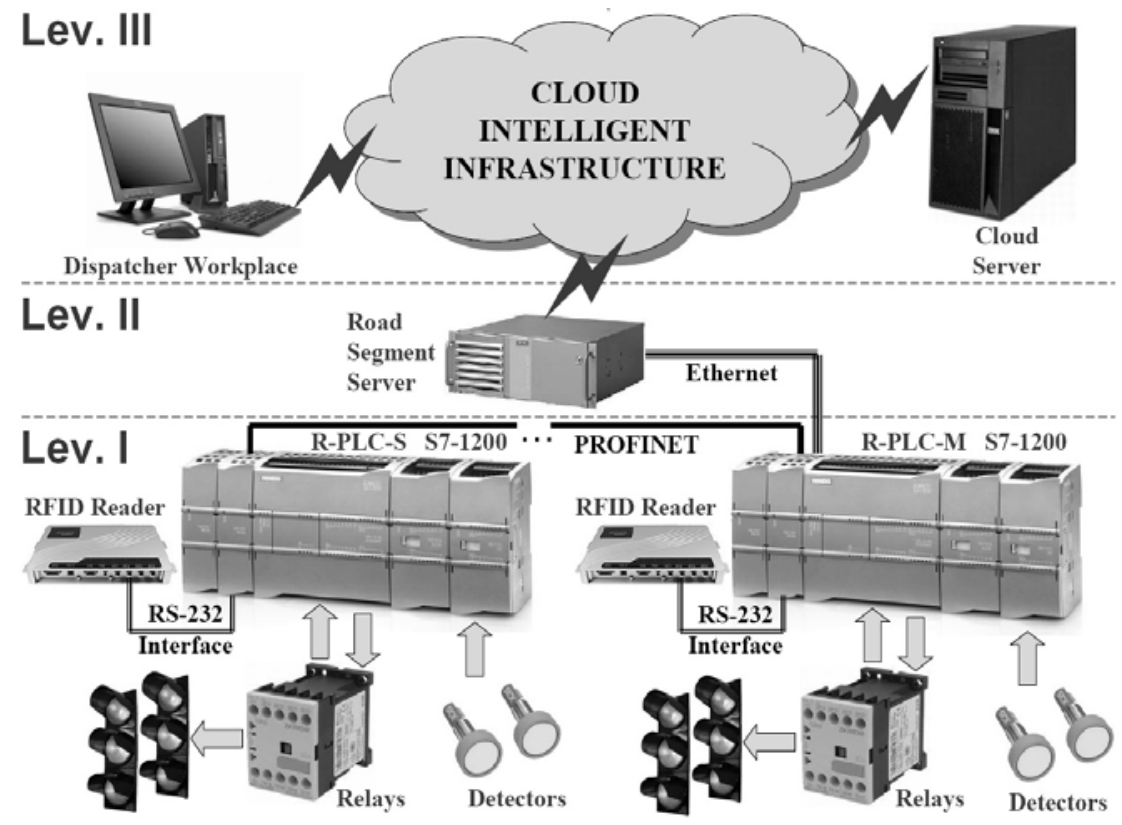

Fig. 4. Architecture of the advanced infrastructure platform of virtual cyberspace of intelligent transport systems. 
The main elements of this architecture are described in detail in $[4,6]$.

Thus, the presented intelligent cloud infrastructure becomes a self-sufficient coordinating supersystem, a special value in such a system is assigned to the adaptive control center, which should become the basis for the formation of intelligent transport systems of a new generation, providing highly adaptive traffic management on the basis of information integration of various heterogeneous subsystems and technical devices [2-4, 13, 14].

\section{Results and Discussion}

Studies [2-4] show that the proposed approach in the field of intelligent traffic management based on fuzzy situational management and virtual cyberspace allows smart and elegant (including in terms of computing time consumption) implementation and analysis of the relationship between the main TF parameters (speed, intensity, density) on many levels [17, $20,21]$ investigation of TF behavior at critical points - saturation and congestion, and also guaranteeing highly adaptive traffic management based on information integration of various heterogeneous subsystems and technical devices.

The proposed scientifically-methodical foundations and software and hardware tools allow solving a rather large range of theoretical and applied problems in the field of transport planning and modeling.

In particular, in terms of creating effective systems of traffic management:

- modelling of existing and forecasted traffic flows;

- analysis and assessment of road traffic rules and intensity;

- planning of effective transport infrastructure;

- scripting "what happens if ";

- creating a platform for operation of situational management centers and decision support systems of various types and assignments.

As to solving theoretical and applied problems of macro- and micro transport process modeling they will provide a mathematical tool for analyzing the contradictions between real data and their reproduction in mathematical models of transport processes, in particular:

- contradictions between dispersion of the traffic parameters actual data and the corresponding relationship of mathematical models;

- the contradiction between the constant value of the ratio of the critical density to the maximum transport flow density for mathematical models and the variable value of this ratio in the real transport flow;

- the contradiction between the uniformity of the relationship form of the transport flow parameters for mathematical models and the change in the functional form for real ratios of intensity-density, speed-density, etc.;

- the contradiction between possible gaps between the values of the transport flow characteristics in transition from a stable state to congestion and smooth relations for theoretical models.

And also the solution of important promising tasks, such as:

- congestion dynamics modelling;

- a sudden transition from free motion to unstable mode;

- solution of theoretical and applied problems of joint use and data exchange between global and local models, etc.

\section{Conclusions}

In conclusion it should be mentioned that the innovative approach proposed in this article is capable of transforming the existing transportation system radically having turned it into a 
highly intelligent environment. It will not only bring mortality and injury rate on roads to nothing but will also increase social mobility, improve environmental situation; it will also give impulse to development of new services in different fields. Moreover, a new type of cars constructed on the basis of the proposed management paradigm can bury a traditional concept of the car and open new unprecedented opportunities and directions.

\section{References}

1. T.I. Mikheeva, Structural and parametric analysis of intelligent transport systems. Samara: SSC RAS, 380 p. (2010)

2. P.M. Klachek, S.I. Koryagin, Applied calculation methods, models and algorithms used in the organization and management of traffic, Monograph. Kaliningrad: Immanuel Kant Baltic Federal University Publishing House, 190 p. (2015)

3. P.M. Klachek, S.I. Koryagin, O.A. Lizorkina, Intelligent system engineering, Monograph. Kaliningrad: Immanuel Kant Baltic Federal University Publishing House, 244 p. (2015)

4. P.M. Klachek, S.I. Koryagin, A.V. Kolesnikov, E.S. Minkova, Hybrid adaptive intelligent systems. Part 1: Theory and technology of development, Monograph. Kaliningrad: Kaliningrad: Immanuel Kant Baltic Federal University Publishing House, 374 p. (2011)

5. V.I. Gnatyuk, The law of optimal construction of technocenoses, 3rd ed., Moscow: "Direct-Media" Publishing House, 476 p. (2014)

6. Yu.V. Belov, O. A. Guz, A.N. Poletaykin, Three-level architecture of the distributed automation system for traffic management, Ensuring safety and comfort of road traffic: problems and solutions: materials of the international scientific and practical conference. Kharkov: Publishing house of Kharkov National Road University, P. 123126 (2011)

7. S. Koryagin, P. Klachek, E. Koryagin, A. Kulakov, The Development Of Hybrid Intelligent Systems On The Basis Of Neurophysiological Methods And Methods Of Multi-Agent Systems, in Conf. Rec. 2016 IEEE int. First International Conference on Data Stream Mining \& Processing, pp. 95-102 (2016)

8. S. Koryagin, P. Klachek, V. Vasileva, Development Of Bionic Approaches In The Microelectromechanical Systems Design Based On Cognitive Knowledge Bank, in Conf. Rec. 2017 IEEE int. 14 International Conference The Experience of designing and application of cad systems in microelectronics , pp. 185-192 (2017)

9. D. Bauman, D. Fierro, Intelligent Transportation System in plain English // Traffic technology international, P. 53-56 (1998)

10. Bergan, A.T., Taylor, W.B., Bushman R. Keep on trucking. Safer commercial traffic with ITS // Traffic technology international. Annual Review, 1998. P. 239-242.

11. D. Gerlough, M. Hueber, Traffic flow theory, A Monograph. Transportation Research Board. Special Report 165, Washington D. C. 220 p., (1999)

12. D. Gibson, P. Ross, Simulation of traffic in street networks, Transp. Eng., 57 (12), pp. 19-27 (1977)

13. P. Gipps, B. Wilson, Multsim, A computer package for simulating mulxLtilane traffic flows. Proc., 4 Biennial Conf., Simulation Soc. Aust., (1980)

14. I. Nuttal, Hunting out the budgets. An informal look at who's spending what ITS. "Traffic technology international", pp. 21-22 (1998) 
15. M Mehdizadeh, M Soleymani, A Hossein, Abolmasoumi Stability control of a road vehicle considering model and parametric uncertainties, in International Journal of Vehicle Autonomous Systems, 13 (3), pp. 203 - 220 (2017)

16. G Büyükyildiz, O Pion, C Hildebrandt, M Sedlmayr, Identification of the driving style for the adaptation of assistance systems, in International Journal of Vehicle Autonomous Systems, 13 (3), pp.244 - 260 (2017)

17. V Jain, T Weiskircher, Prediction-based hierarchical control framework for autonomous vehicles, in International Journal of Vehicle Autonomous Systems, 12 (45), pp.307 - 333 (2014)

18. M Khalfaoui, K Hartani, M Merah, N Aouadj, Development of shared steering torque system of electric vehicles in presence of driver behaviour estimation, in International Journal of Vehicle Autonomous Systems, 14 (1), pp.18 - 39 (2018)

19. $\mathrm{H} \mathrm{Lu}, \mathrm{Q} \mathrm{Liu}, \mathrm{Y}$ Shi, F Yu, Estimation of vehicle sideslip angle and individual tyreroad forces based on tyre friction circle concept, in International Journal of Vehicle Autonomous Systems, 14 (4), pp. 274 - 305 (2017)

20. Y Lue, S Chang Non-linear dynamics and control of an automotive suspension system based on local and global bifurcation analysis, in International Journal of Vehicle Autonomous Systems, 13 (4), pp.340 - 359 (2017)

21. M Mehdizadeh, M Soleymani, A Hossein, Abolmasoumi Stability control of a road vehicle considering model and parametric uncertainties, in International Journal of Vehicle Autonomous Systems, 13 (3), pp.203 - 220 (2017) 